\title{
$\beta$-Sheet peptide-assisted polymerization of diacetylene at the air-water interface and thermochromic property
}

\author{
Tomoyuki Koga, Tomohisa Taguchi and Nobuyuki Higashi
}

A peptide-diacetylene hybrid (PDH), in which 5,7-dodecadiynedioic acid was conjugated to the amphiphilic penta-peptide Leu-Lys-Leu-Lys-Leu, which can form $\beta$-sheets on both ends of the diacetylene moiety, was prepared through solid-phase peptide synthesis using fluorenylmethoxycarbonyl chemistry. Spreading experiments and spectroscopic observations showed that the PDH formed a stable Langmuir monolayer, primarily in the parallel $\beta$-sheet conformation at the air-water interface. When the PDH monolayer was exposed to ultraviolet radiation and a single-layer Langmuir-Blodgett (LB) film of the treated monolayer was deposited on a $\mathrm{CaF}_{2}$ plate, the polymerization proceeded successfully and provided highly $\pi$-conjugated, blue-colored polydiacetylenes with absorption maxima at 653 and $640 \mathrm{~nm}$, respectively. The polymerized PDH LB film displayed almost completely reversible thermochromism between blue and red (less conjugated) states upon heating and cooling.

Polymer Journal (2012) 44, 195-199; doi:10.1038/pj.2011.105; published online 9 November 2011

Keywords: $\beta$-Sheet; diacetylene; peptide; surface monolayer; thermochromism; topochemical polymerization

\section{INTRODUCTION}

Polydiacetylenes are conjugated polymeric materials with unique optical properties. The efficient polymerization of diacetylene yielding highly conjugated backbones requires the positioning of atoms in a crystalline lattice, and there is little displacement before and after polymerization; such a reaction is known as a topochemical reaction. ${ }^{1-3}$ When properly aligned, diacetylene groups undergo ultraviolet (UV)-induced photopolymerization to form an ene-yne alternating polymer backbone, resulting in organized structures. In aqueous environments, these materials often appear blue or red, with the blue color indicating greater order in the conjugated $\pi$-system. ${ }^{4}$ As the conjugated $\pi$-system becomes less ordered, the observed color shifts from blue to red. This unique chromatic property has made polydiacetylenes applicable to colorimetric biosensors. ${ }^{5-11}$

We describe herein the thermoreversible color change of polydiacetylene prepared through $\beta$-sheet peptide-templated polymerization at the air-water interface. $\beta$-Sheet peptides are promising candidates for such a template or scaffold because they are often present in natural fibers as a structural element. Langmuir monolayers of $\beta$-sheet peptides were, for example, reported to function as templates and serve as nuclei for the formation of inorganic nanocrystallites at the air-water interface. ${ }^{12}$ Recently, numerous research groups have demonstrated that amphiphilic peptides self-assemble into $\beta$-sheet nanofiber architectures, ${ }^{13-20}$ the surface of which has potential as a novel nanotemplate. ${ }^{21-23}$ Several groups ${ }^{24-27}$ have separately used $\beta$-sheet peptide nanofibers as templates to organize diacetylenes or dienes and promote their topochemical polymerization. In this study, we used Langmuir monolayers at the air-water interface and $\beta$-sheetforming oligopeptides to align diacetylene groups one-dimensionally, promoting facile topochemical polymerization and stabilizing the resultant well-conjugated polydiacetylenes. In addition, if the Langmuir monolayers could be transferred onto an appropriate solid plate using an Langmuir-Blodgett (LB) technique, one-dimensional alignment of diacetylene moieties would also be accomplished, and then anisotropically oriented polydiacetylene thin films could be produced by photopolymerization. Such films have potential applications in electron-conducting nanodevices such as perfect quantum wires. ${ }^{28}$ We designed and synthesized a peptide-diacetylene hybrid (PDH), which includes (1) a penta-peptide segment composed of an amphiphilic alternating Leu-Lys sequence to induce anisotropic self-assembly through the formation of a $\beta$-sheet structure at the air-water interface; (2) a symmetric structure containing two penta-peptide segments at the ends to enhance the hydrogen bonding network; and (3) a diacetylene moiety integrated directly into the $\beta$-sheet guides to promote the appropriate alignment for topochemical polymerization and to stabilize the highly conjugated structure of the resultant polydiacetylene. A PDH derivative lacking a peptide segment at one terminus (PDH-ot) was also synthesized and used for comparison.

\section{EXPERIMENTAL PROCEDURE}

Synthesis of peptide-diacetylene hybrids (PDH and PDH-ot) The PDH and the PDH-ot were prepared by solid-phase peptide synthesis using fluorenylmethoxycarbonyl chemistry. The peptide segment was first synthesized on a CLEAR (crosslinked ethoxylateacrylate resin, Peptide Institute, Osaka, Japan) using fluorenylmethoxycarbonyl-Leu and fluorenylmethoxycar- 
bonyl-Lys (3 equiv), 1-hydroxy-7-azabenzotriazole (3 equiv) and 1,3-diisopropylcarbodiimide (3 equiv) in dimethylformamide for coupling and piperidine (25\%)/dimethylformamide for fluorenylmethoxycarbonyl removal. The peptide was then coupled to 5,7-dodecadiynedioic acid (Nacalai Tesque, Kyoto, Japan) in the same manner. To cleave the diacetylene-containing peptide from the resin and remove the side-chain protecting groups, the peptide resin was treated with trifluoroacetic acid/ $\mathrm{CH}_{2} \mathrm{Cl}_{2}(9 / 1 \mathrm{v} / \mathrm{v})$. All peptides were separated and purified by reversed-phase high-performance liquid chromatography (GE Healthcare, Uppsala, Sweden, ÄKTA explore 10S) on a COSMOSIL 5C18-AR-II column $(10 \times 250 \mathrm{~mm}$, Nacalai Tesque) using a linear gradient of wateracetonitrile containing $0.05 \%$ trifluoroacetic acid. The peptides were identified using matrix-assisted laser desorption ionization-time of flight MS (MALDITOF MS; Shimadzu Biotech Axima CFR plus, Shimadzu, Kyoto, Japan). Before high-performance liquid chromatography purification, there were two major peaks in the MALDI-TOF MS spectrum that were assigned to the PDH and its derivative lacking a peptide segment at one terminus (PDH-ot). Separation and purification by high-performance liquid chromatography were successfully completed using the same system. (MALDI-TOF MS: observed $[\mathrm{M}+\mathrm{H}]^{+} \mathrm{PDH}$ : $m / z ; 1471.8$ (theoretical: 1471.0), observed $[\mathrm{M}+\mathrm{H}]^{+} \mathrm{PDH}$-ot: $\mathrm{m} / z ; 860.9$ (theoretical: 860.1).

\section{Spreading experiments}

Monolayers were obtained by spreading a benzene/trifluoroethanol $(7 / 3 \mathrm{v} / \mathrm{v})$ solution (approximately $1 \mathrm{mg} \mathrm{ml}^{-1}$ ) of the $\mathrm{PDH}$ or the PDH-ot on water that was purified $(\rho>18 \mathrm{M} \Omega \mathrm{cm})$ using an Arium $611 \mathrm{VF}$ system (Sartorius, Göttingen, Germany). Fifteen minutes after spreading, the monolayer was compressed continuously at a rate of $0.25 \mathrm{~cm}^{2} \mathrm{~s}^{-1}$. The Wilhelmy plate method and a Teflon-coated trough with a FSD-220 microprocessor-controlled film balance (USI system, Fukuoka, Japan) with a precision of $0.01 \mathrm{mN} \mathrm{m}^{-1}$ were used for surface pressure measurements. LB films were prepared by transferring the corresponding monolayers onto a $\mathrm{CaF}_{2}$ or mica plate at a constant surface pressure via a vertical dipping method.

\section{Polymerization and measurements}

Polymerization of the monolayers and the LB films was induced by irradiating the samples at $254 \mathrm{~nm}$ using a compact handheld 4-W UV lamp. Samples were irradiated at $10 \mathrm{~cm}$ from the light source. The polymerization process was monitored by reflection-absorption spectroscopy with a MCD-100 multichannel photodetector (Otsuka Electronics, Osaka, Japan) or by UV-visible spectroscopy with a UV-2100 system (Shimadzu). Attenuated total reflection Fourier transform infrared spectroscopy (FTIR) spectra and circular dichroism
(CD) spectra of the LB films were recorded using a Nexus 470 FTIR with an MCT detector (Thermo Nicolet, Madison, WI, USA) and a J-805 CD spectrophotometer (JASCO, Tokyo, Japan), respectively. Atomic force microscopy (AFM) images were collected at ambient temperature using a NanoscopeIIIa (Digital Instruments, Santa Barbara, CA, USA) operated in tapping mode using a silicon tip (tip radius, $10 \mathrm{~nm}$ ). The scanning speed was a line frequency of $1 \mathrm{~Hz}$, and the original images were sampled at a resolution of $512 \times 512$ points.

\section{RESULTS AND DISCUSSION}

The surface pressure-area isotherms for the amphiphilic PDH at the air-water interface are shown in Figure 2a. The compression of the PDH resulted in a surface pressure-area isotherm that exhibited a liftoff at approximately $4.5 \mathrm{~nm}^{2}$ per molecule and a knee in the region of 4.5$3.5 \mathrm{~nm}^{2}$ per molecule, indicating a transition from a liquid-expanded phase to a liquid-condensed phase. Further compression caused an abrupt increase in the surface pressure from 5 to $23 \mathrm{mN} \mathrm{m}^{-1}$, which corresponded to a compression from 3.5 to $1.8 \mathrm{~nm}^{2}$ per molecule. The Langmuir monolayer was observed to collapse at $23 \mathrm{mN} \mathrm{m}^{-1}$, at which the occupied molecular area was $1.8 \mathrm{~nm}^{2}$ per molecule. This value is consistent with the $\left(1.81 \mathrm{~nm}^{2}\right.$ per molecule) value predicted by assuming that the peptide segments form an extended $\beta$-sheet structure and that the $\beta$-strands align parallel to the air-water interface (Figure 1b).

The stability of the Langmuir monolayer at the air-water interface was examined by submitting the monolayer to compression/expansion cycles. Figure $2 \mathrm{~b}$ shows the compression/expansion cycles up to $\mathrm{a}$ surface pressure of $20 \mathrm{mN} \mathrm{m}^{-1}$. Four successive compression/expansion cycles were completed. The behavior of the monolayer during these cycles demonstrated that the monolayer did not reach the collapse surface pressure, and thus a stable monolayer was formed on pure water in this range of compression. It should be noted that hysteresis behavior in the compression/expansion isotherms was observed only during the first cycle, suggesting a strong interaction between the peptide segments (conformational transition) induced by compression.

To confirm the secondary structure of the peptide segments, CD and FTIR spectroscopic analyses were performed for the single-layer LB film on a $\mathrm{CaF}_{2}$ plate with an upward stroke at different surface pressures. Figure 3 shows the CD and FTIR spectra recorded for the LB films deposited from the PDH Langmuir monolayer. For the LB film prepared at $5 \mathrm{mN} \mathrm{m}^{-1}$, the $\mathrm{CD}$ spectrum gave a broad negative<smiles>CC(C)CC(NC(=O)C(CCCCN)NC(=O)C(CC(C)C)NC(=O)C(CCCCN)NC(=O)C(CC(C)C)NC(=O)CCC#CCCC(=O)NC(CC(C)C)C(=O)NC(CCCCN)C(=O)NC(CC(C)C)C(=O)NC(CCCCN)C(=O)NC(CC(C)C)C(=O)O)C(=O)O</smiles>

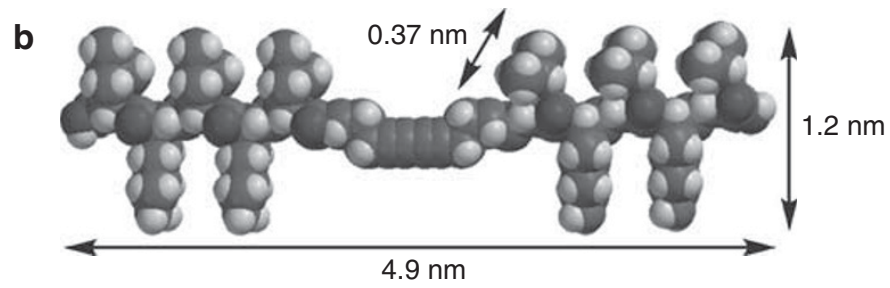

Figure 1 Chemical structure (a) and its corresponding space-filling molecular model (b) of peptide-diacetylene hybrid (PDH). A full color version of this figure is available at Polymer Journal online. 

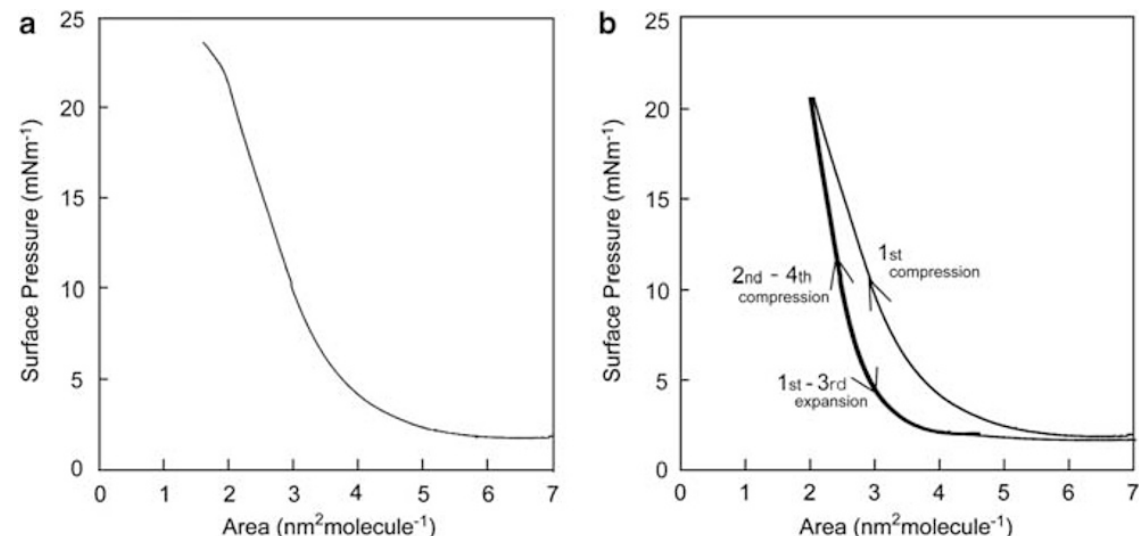

Figure 2 Surface pressure-area isotherms of the peptide-diacetylene hybrid (PDH) monolayer (a) and the compression/expansion cycles (b) on pure water at $\mathrm{pH} 5.8$ and $20^{\circ} \mathrm{C}$.
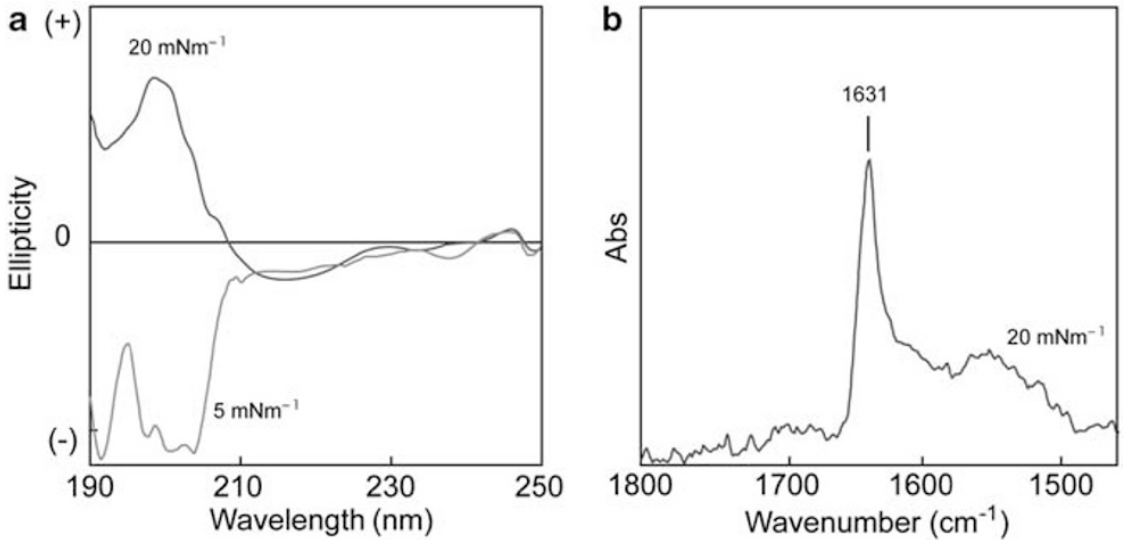

Figure 3 (a) Circular dichroism (CD) spectra of the peptide-diacetylene hybrid (PDH) Langmuir-Blodgett (LB) films prepared at $5 \mathrm{mN} \mathrm{m}^{-1}$ and $20 \mathrm{mN} \mathrm{m}^{-1}$,

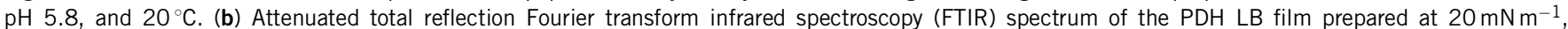
$\mathrm{pH} 5.8$, and $20^{\circ} \mathrm{C}$. Every sample was measured as a single-layer LB film on a $\mathrm{CaF}_{2}$ plate deposited using the vertical dipping method at the indicated surface pressures. A full color version of this figure is available at Polymer Journal online.

peak at approximately $200 \mathrm{~nm}$, suggesting a random coil conformation (Figure 3a). Compression of the monolayer up to $20 \mathrm{mN} \mathrm{m}^{-1}$ was found to induce the formation of $\beta$-sheet structure, as demonstrated by the appearance of negative and positive peaks at approximately 216 and $194 \mathrm{~nm}$, respectively. The FTIR spectrum also supports the conclusions based on the CD spectrum; that is, the PDH LB film prepared at $20 \mathrm{mN} \mathrm{m}^{-1}$ exhibited a peak at $1631 \mathrm{~cm}^{-1}$ in the amide I band region assigned to the $\beta$-sheet conformation. Additional information about the $\beta$-sheet structure was revealed by this FTIR spectrum; there was no remarkable peak at $1690 \mathrm{~cm}^{-1}$ due to an anti-parallel $\beta$-sheet structure, ${ }^{29}$ indicating that the peptide segments in the PDH LB film deposited at $20 \mathrm{mN} \mathrm{m}^{-1}$ form a primarily parallel $\beta$-sheet structure; however, the existence of a minor anti-parallel component cannot be excluded.

Figure 4a displays an AFM image of a PDH LB film deposited onto a mica plate at a surface pressure of $20 \mathrm{mN} \mathrm{m}^{-1}$. One-dimensionally grown, fibrous objects are clearly observed, and their widths varied. Interestingly, the fiber axes were aligned almost parallel to the lifting direction of the mica plate during deposition. The fiber height was estimated to be $1.1 \pm 0.2 \mathrm{~nm}$, which is in fair agreement with the molecular thickness of the PDH $(1.2 \mathrm{~nm})$ calculated by assuming that the peptide segments form a $\beta$-sheet structure (Figure $1 \mathrm{~b}$ ). The AFM image showed a small cavity between the fibrous objects. The LB film was prepared at a surface pressure of $20 \mathrm{mN} \mathrm{m}^{-1}$, at which the mean occupied molecular area was calculated to be $2.1 \mathrm{~nm}^{2}$ per molecule from the surface pressure-area curve in Figure 2a. This value is somewhat larger than that $\left(1.8 \mathrm{~nm}^{2}\right)$ for the molecular size from Figure $1 \mathrm{~b}$. This difference seems to explain why such a cavity was generated in the LB film. Figure $4 \mathrm{~b}$ displays the proposed structural model of the PDH monolayer at the air-water interface, in which the side chains of Lys and Leu face the water phase and the air phase, respectively, stabilizing the parallel $\beta$-sheet structure. As a result of this conformation, the diacetylene moieties must be aligned one dimensionally. However, the co-existence of a bundled fibrous structure based on anti-parallel $\beta$-sheets cannot be excluded based on the FTIR data and the AFM observations.

The in situ photopolymerization of the PDH monolayer was investigated using $254 \mathrm{~nm}$ UV light as the source of radiation. A reflectionadsorption spectroscopy system equipped with a Langmuir trough was used to follow the photopolymerization process. We did not observe any band formation for the monolayer at a surface pressure of $5 \mathrm{mN} \mathrm{m}^{-1}$ upon irradiation. This result indicates that the diacetylene moieties were not close enough to initiate photopolymerization because the peptide segments were in the random coil 

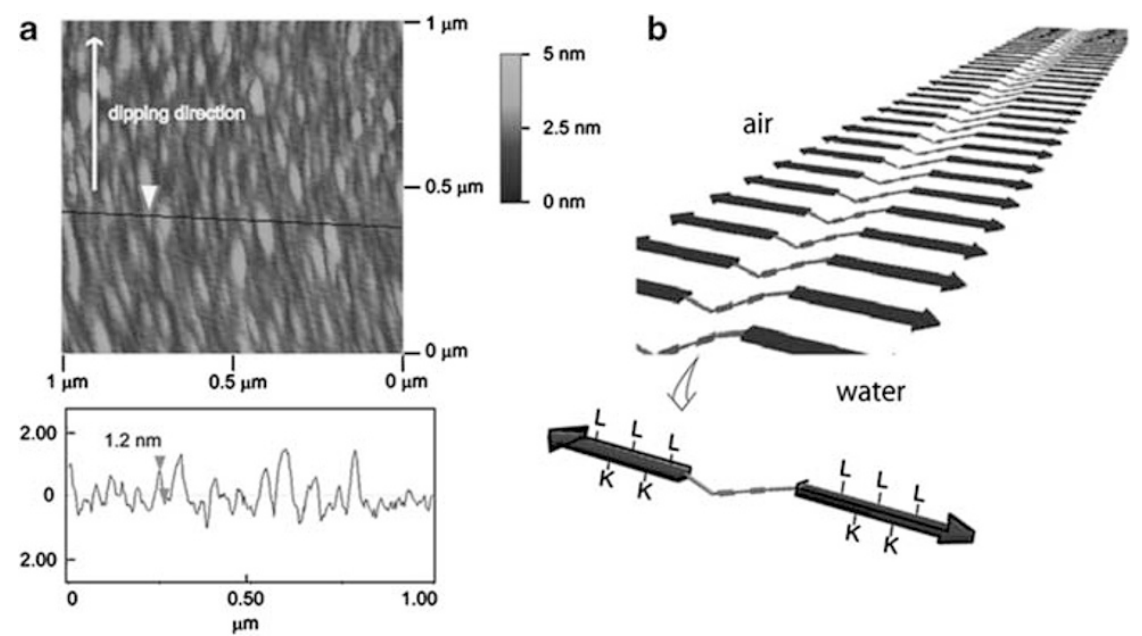

Figure 4 (a) Tapping-mode atomic force microscopy (AFM) image of a single-layer peptide-diacetylene hybrid (PDH) Langmuir-Blodgett (LB) film on mica

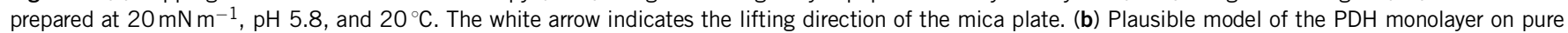
water:one-dimensional alignment of the diacetylene moiety guided by $\beta$-sheet peptides. A full color version of this figure is available at Polymer Journal online.
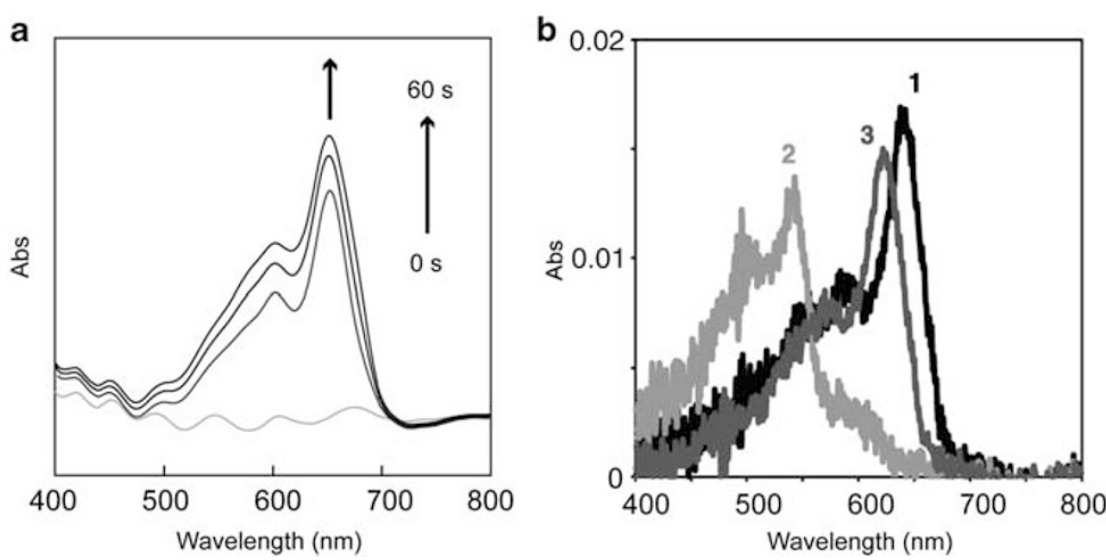

Figure 5 (a) Change in the absorption spectrum of the peptide-diacetylene hybrid (PDH) monolayer on pure water upon ultraviolet irradiation at a constant

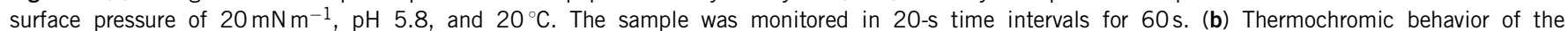
polymerized PDH Langmuir-Blodgett (LB) film dipped in pure water: curves $1,10^{\circ} \mathrm{C} ; 2,68^{\circ} \mathrm{C} ; 3$, after cooling back to $10^{\circ} \mathrm{C}$. A full color version of this figure is available at Polymer Journal online.

conformation at this low surface pressure. Compression of the monolayer up to a surface pressure of $20 \mathrm{mN} \mathrm{m}^{-1}$ yielded a bluecolored polymer monolayer upon irradiation that could also be observed with the naked eye. The molecular area of the PDH monolayer did not exhibit a significant change after UV irradiation at a constant surface pressure of $20 \mathrm{mN} \mathrm{m}^{-1}$. These results imply that the topochemical polymerization of the diacetylene moieties progressed rapidly. The UV-visible absorption was monitored for $60 \mathrm{~s}$, as shown in Figure 5a. The peak at $653 \mathrm{~nm}$ is characteristic of the so-called blue state of polydiacetylene. Interestingly, there was no significant band at $530-550 \mathrm{~nm}$ (red state). Although there is still discussion as to whether such long-wavelength absorption depends on a solid-state effect, ${ }^{30}$ the observed rapid polymerization and formation of blue-state polydiacetylene in this study was due at least in part to the one-dimensional organization of the diacetylene moieties guided by the formation of $\beta$-sheet peptides and the two-dimensional organization at the air-water interface (Figure $4 \mathrm{~b}$ ). In fact, the distance between parallel $\beta$-sheets $(\sim 5 \AA)$ is consistent with the preferred distance between diacetylene monomers $(4.7 \sim 5.2 \AA)^{3,30}$ for efficient topochemical polymerization.
For comparison, the same polymerization experiments were performed using the PDH-ot, which has a peptide segment only at one terminus. The PDH-ot formed stable monolayers on water under the same conditions as the PDH did, but the photopolymerization did not proceed at any surface pressure. The FTIR spectrum of the deposited $\mathrm{LB}$ film of the $\mathrm{PDH}$-ot prepared at $20 \mathrm{mN} \mathrm{m}^{-1}$ revealed that the peptide segments formed primarily anti-parallel $\beta$-sheets. When the $\mathrm{PDH}$-ot molecules are in the anti-parallel $\beta$-sheet conformation at the two-dimensional interface, the distance between the diacetylene moieties was estimated to be twice that in the parallel $\beta$-sheet conformation, and this greater distance is definitely a disadvantage in the topochemical reaction of diacetylene moieties. ${ }^{31}$

When we tried to deposit polymerized PDH monolayers onto solid plates using the vertical dipping method, the monolayers were not transferred onto any substrate, probably because of the stiffness of the polymer. Therefore, unpolymerized PDH monolayers were first transferred to the substrates at a surface pressure of $20 \mathrm{mN} \mathrm{m}^{-1}$, and then photopolymerization was performed. The polymerization proceeded and provided a blue-state polymer with an absorption maximum at 
$640 \mathrm{~nm}$, whereas it is somewhat shorter than that $(653 \mathrm{~nm})$ of the polymer prepared in the Langmuir monolayer state. Therefore, the results again demonstrated that the molecular order in the Langmuir monolayer state, which is proper for topochemical polymerization of diacetylene moieties, was maintained even in the LB film state with the support of $\beta$-sheet network.

Finally, thermochromic behavior of the polymerized LB film was examined spectroscopically. Figure $5 \mathrm{~b}$ exhibits the absorption spectral change of the LB film dipped in pure water upon heating. When the temperature was raised from 10 to $68^{\circ} \mathrm{C}$, the peak at $640 \mathrm{~nm}$ (blue state) disappeared and that at $530 \mathrm{~nm}$ (red state) significantly increased. Reversibly, lowering the temperature to $10^{\circ} \mathrm{C}$ returned the spectrum to the original one (blue state), whereas the absorption maximum shifted slightly to the shorter wavelength $(630 \mathrm{~nm})$. Such a reversible thermochromism should be noted. Previously, we have demonstrated the photopolymerization of a boronic acid-containing diacetylene lipid monolayer and the thermochromism. ${ }^{11}$ In this case, a fully thermochromic behavior was surely observed, but the blue polymer could not completely convert to the red one because the boronic acid moieties spontaneously dehydrated and formed a polymeric borate glass that would inhibit the color transition. Therefore, the thermochromism observed in this study is of interest and provide an important insight to develop a novel nanomaterial such as highly sensitive sensing devices. The $\beta$-sheet peptides attached on both ends of diacetylene moiety have a key role to make the topochemical polymerization easier and to offer reversibly color-changeable conjugated backbones, based on hydrogen bonding network.

\section{CONCLUSION}

We successfully synthesized novel PDHs, which have a $\beta$-sheet-forming peptide segment at both termini (PDH) and one terminus (PDHot) of the diacetylene moiety. They formed stable Langmuir monolayers at neutral $\mathrm{pH}$ (5.8). Circular dichroism and FTIR spectroscopic data demonstrated that the PDH and PDH-ot LB films included primarily the parallel $\beta$-sheet conformation and the anti-parallel $\beta$-sheet conformation, respectively. The AFM observation for the PDH LB film proved the existence of one-dimensionally grown, fibrous objects and their parallel alignment with the lifting direction of the plate during deposition. Upon UV irradiation, the PDH monolayer exhibited a rapid polymerization following topochemical process and provided a highly conjugated blue polymer, reflecting well-ordered, one-dimensional alignment of diacetylene moieties assisted with the parallel $\beta$-sheet network. On the other hand, in the PDH-ot monolayer, the photopolymerization was completely inhibited because of too great distance between polymerizable moieties to react. The polymerized PDH blue film exhibited almost completely reversible thermochromism between blue and red states by an elastic template effect of the $\beta$-sheet network on conjugated polydiacetylene backbones. From these results, it can be concluded that $\beta$-sheet-forming peptide segments attached on both sides of diacetylene moiety and the two-dimensional molecular system including the LB deposition process have key roles in the efficient topochemical polymerization, giving a highly conjugated blue polymer, the controlled alignment of the resultant polydiacetylene and also its reversible thermochromic behavior.

\section{ACKNOWLEDGEMENTS}

This work was supported in part by a grant-in-aid for young scientific research (No. 21750130) from the Ministry of Education, Culture, Sports, Science and Technology (MEXT) Japan, and by the Project on 'Creating Research Center for Advanced Molecular Biochemistry' from the MEXT.
1 Cantov, H.- J. Polydiacetylenes, Vol. 63Springer-Verlag, Berlin, 1984.

2 Fouassier, J. P., Tieke, B. \& Wegner, G. Photochemistry of the polymerization of diacetylenes in multilayers. Isr. J. Chem. 18, 227-232 (1979).

3 Tieke, B., Lieser, G. \& Wegner, G. Polymerization of diacetylenes in multilayers. J. Polym. Sci., Part A: Polym. Chem. 17, 1631-1644 (1979).

4 Huo, Q., Wang, S. P., Pisseloup, A., Verma, D. \& Leblanc, R. M. Unusual chromatic properties observed from polymerized dipeptide diacetylenes. Chem. Commun. 1601-1602 (1999).

5 Charych, D. H., Nagy, J. O., Spevak, W. \& Bednarski, M. D. Direct colorimetric detection of a receptor-ligand interaction by a polymerized bilayer assembly. Science 261, 585-588 (1993).

6 Reichert, A., Nagy, J. O., Spevak, W. \& Charych, D. H. Polydiacetylene liposomes functionalized with sialic acid bind and colorimetrically detect influenza virus. J. Am. Chem. Soc. 117, 829-830 (1995).

7 Jelinek, R. Colorimetric sensors for drug discovery and biomedical diagnostics. Dev. Res. 50, 497-501 (2000).

8 Kolusheva, S., Kafri, R., Katz, M. \& Jelinek, R. Cation-selective color sensors composed of ionophore-phospholipid-polydiacetylene mixed vesicles. J. Am. Chem. Soc. 122, 776-780 (2000).

9 Song, J., Cisar, J. S. \& Bertozzi, C. R. Functional self-assembling bolaamphiphilic polydiacetylenes as colorimetric sensor scaffolds. J. Am. Chem. Soc. 126, 8459-8465 (2004).

10 Kuo, T. \& O'Brien, D. F. Synthesis and properties of diacetylenic glutamate lipid monomer and polymer: thermochromic polydiacetylene free-standing films. Macromolecules 23, 3225-3230 (1990).

11 Niwa, M., Shibahara, S. \& Higashi, N. Diacetylenic monolayers containing a boronic acid moiety form a chemically and thermally stable poly(diacetylene) film on water. J. Mater. Chem. 10, 2647-2651 (2000).

12 Bekele, H., Fendler, J. H. \& Kelly, J. W. Self-assembling peptidomimetic monolayer nucleates oriented CdS nanocrystals. J. Am. Chem. Soc. 121, 7266-7267 (1999).

13 Zhang, S., Holmes, T., Lockshin, C. \& Rich, A. Spontaneous assembly of a selfcomplementary oligopeptide to form a stable macroscopic membrane. Proc. Natl Acad. Sci. USA. 90, 3334-3338 (1993).

14 Takahashi, Y., Ueno, A. \& Mihara, H. Design of a peptide undergoing $\alpha-\beta$ structural transition and amyloid fibrillogenesis by the introduction of a hydrophobic defect. Chem. Eur. J. 4, 2475-2484 (1998).

15 Lashuel, H. A., LaBrenz, S. R., Woo, L., Serpell, L. C. \& Kelly, J. W. Protofilaments, filaments, ribbons, and fibrils from peptidomimetic self-assembly: implications for amyloid fibril formation and materials science. J. Am. Chem. Soc. 122, 5262-5277 (2000).

16 Hartgerink, J. D., Beniash, E. \& Stupp, S. I. Self-assembly and mineralization of peptide-amphiphile nanofibers. Science 294, 1684-1688 (2001).

17 Murasato, K., Matsuura, K. \& Kimizuka, N. Self-assembly of nanofiber with uniform width from wheel-type trigonal- $\beta$-sheet forming peptide. Biomacromolecules 9, 913-918 (2008).

18 Koga, T., Matsuoka, M. \& Higashi, N. Structural control of self-assembled nanofibers by artificial $\beta$-sheet peptides composed of D- or L-isomer. J. Am. Chem. Soc. 127, 17596-17597 (2005).

19 Koga, T., Higuchi, M., Kinoshita, T. \& Higashi, N. Controlled self-assembly of amphiphilicoligopeptides into shape-specific nanoarchitectures. Chem. Eur. J. 12, 1360-1367 (2006).

20 Koga, T., Kitamura, K. \& Higashi, N. Enzymatically triggered self-assembly of poly(ethylene glyco)-attached oligopeptides into well-organized nanofibers. Chem. Commun 4897-4898 (2006)

21 Kodama, H., Matsumura, S., Yamashita, T. \& Mihara, H. Contraction of a protein array on amyloid-like fibrils using co-assembly of designed peptides. Chem. Commun 2876-2877 (2004).

22 Li, L. \& Stupp, S. I. One-dimensional assembly of lipophilic inorganic nanoparticles templated by peptide-based nanofibers with binding functionalities. Angew. Chem. Int. Ed. 44, 1833-1836 (2005).

23 Koga, T., Murakami, R. \& Higashi, N. Surface modification of peptide nanofiber by using antigen-antibody interaction. Trans. Mater. Res. Soc. Jpn. 32, 371-374 (2007).

24 Jahnk, E., Lieberwirth, I., Severin, N., Rabe, J. P. \& Frauenrath, H. Topochemical polymerization in supramolecular polymers of oligopeptide-functionalized diacetylenes. Angew Chem Int Ed 45, 5383-5386 (2006).

25 Hsu, L., Cvetanovich, G. L. \& Stupp, S. I. Peptide amphiphile nanofibers with conjugated polydiacetylene backbones in their core. J. Am. Chem. Soc. 130, 3892-3899 (2008).

26 Van den Henvel, M. Löwik, D. W. P. M. \& vanHest, J. C. M. Self-assembly and polymerization of diacetylene-containing peptide amphiphiles in aqueous solution. Biomacromolecules 9, 2727-2734 (2008).

27 Mori, T., Yasutake, S., Inoue, H., Minagawa, K., Tanaka, M., Niidome, T. \& Katayama, Y "Threading" of $\beta$-sheet peptides via radical polymerization. Biomacromolecules $\mathbf{8}$, 318-321 (2007).

28 Schott, M. in Photophysics of Moleculat Materials. From Single Molecules to Single Crystals (ed. Lanzani, G.) 49-151 (Wiley-VCH, Berlin, 2006).

29 Miyazawa, T. \& Blout, E. R. The infrared spectra of polypeptides in various conformations: amide I and II bands. J. Am. Chem. Soc. 83, 712-719 (1961).

30 Schott, M. The colors of polydiacetylenes: a commentary. J. Phys. Chem. B 110, 15864-15868 (2006).

31 Baughman, R. H. \& Yee, K. C. Solid-state polymerization of linear and cyclic acetylenes. J. Polym. Sci., Macromol. Rev. 13, 219-239 (1978). 